\title{
Detailed analysis on the new usage of Lei Pu Qing-hong
}

\author{
School of Humanities \& Social Sciceces \\ North China Electric Power University \\ Beijing, P.R. China \\ qhpu@ncepu.edu.cn
}

\begin{abstract}
With the advance of society and increasing versatility of social life, everyday language will inevitably be influenced. As a consequence, new words and new phrases are frequently created; existing words (phrases) are often twisted to express new meaning. While this language tendency brings up interest among linguist, it is worth to analyses the phenomenon in detail from the linguistic perspective. This article explores the meaning, semantics, and generalization of Lei which recently became a catchword.
\end{abstract}

Keywords: Lei, Generalization, semantics.

\section{Introduction}

Since 2008, "Lei" has become a catchword with very high frequency of occurrences in news-papers, magazines, Internet, daily speaking, and other sort of media, such as "Lei-ren"、 "Lei-dao" ect. which were considered to comprise Lei family phrases. Many of them even directly hit media titles, for example "The catchwords are Hen-lei (striking) this summer" in "Sanqing cosmopolitan Newspaper" (07-13-2008), "It is very Lei-ren (ridiculous) to ask leaders to have the lead in taking day off just for shopping" in "Yangtze evening newspaper" (01/16/2009), "The lines are Hen-lei (shocking); the martial arts are amazing" in "Beijing youth newspaper" (07/11/2009), "Feeling democratic new sense from Lei-ren (astonishing) speech of consulting council members" in "Beijing youth newspaper" (01/20/2009), "Lei-dao-le (Shocked) by patriotic consumption" in "China net" (01/13/2009), "Lei-zhao-le (Shocked) by dancers" in "Beijing youth news paper" (11/20/2008), "Lei-dao-tian-wang(King of shocking)" in "Beijing youth news paper" (11/15/2008), "Audience rating champion, the Lei(amazing) name" in "Beijing youth newspaper" (01/16/2009), and much more. In Google search, there are 11,900,000 items for Lei-ren, 757,000 for Lei-dao, and 41,100,000 for Hen-lei. It is hard to ignore this fact that these phrases are so frequently used.

\section{The meaning and its generalization of "Lei"}

In modern Chinese dictionary, Lei refers a loud rumbling heard after a lightning flash due to the expansion of rapidly heated air. It is a noun. However, the character Lei used in catchphrases which are appearing in Internet and other medias, such as Lei, Lei-ren , Lei-dao , Hen-Lei ect, is characterized of ancient Chinese. It can be nouns, verbs, or adjectives. Its meaning varies in different situation and the property changes accordingly. After analysis of Lei phrases appearing in all sorts of media, we noticed that the new meanings directly derived from Lei can be classified as follows:

(1) Terrified, scared like in the situation of stepping on a mine. It is a derogatory term. For example:

a. 'Lei (shocking) you without any hesitation in 2008,' counting the catchwords in this years (Beijing Youth Newspaper, 01-21-2009)

b. 'Champion of viewing rate, you are called Lei': the more blaming and cursing it is getting, the more popular the show is becoming. As for this phenomenon, many professionals think that with viewing rate as its only goal the current broadcasting system for TV show has obvious defects. This leaded to thriving of Lei (shocking) TV shows in 2008 which was ending with the ridiculous result. (Beijing Youth Newspaper, 12-01-2008)

c. Before the action of movie car chasing fully Lei (shocked) audience, there is long way to go and many more car chasings will come in. (Beijing Youth Newspaper, 12-30-2008)

d. This specialist fully Lei (shocked) me (Beijing Youth Newspaper, 12-03-2008)

(2) Shocked, astonished, dumbfound, hard to image. It is a neutral term.

e. The scene where the driver is driving while he is on a drip really Lei (shocked) the police person. (Beijing Youth Newspaper, 01-15-2009)

f. Before get Lei (astonished), go and see what is going on. Xinmo is well known initially for manually manufacturing leather note books. This time it involves in producing lamps of creative shapes. (Beijing Youth Newspaper, 12-17-2008)

g. Using all sorts of rare and bizarre, unimaginable (Lei) tools and letting performer to conduct all kinds of weird 
poses, Xuke seemed to be satisfied with his creativity. (Beijing Youth Newspaper, 12-17-2008)

\section{(3) Funny, ridiculous, anomalous, neither fish nor fowl. A derogatory term.}

h. In the second half, there may be more anomalous (Leiren) lines. Perhaps, this non-historic, but truly realistic lines are what $\mathrm{Wu}$ Yiseng wanted. (Beijing Youth Newspaper, 12-11-2008)

i. Those who were known as video manipulating professionals of so called non-responsibility for being shocked to death (Lei-si-ren-bu-chang-ming) employed fine or immature technologies tirelessly to produce huge amount of funny videos. (New Instant Paper, 05-07-2008)

j. Shocking (Lei-ren) style, wit-lacking plot, annoying advertisement, astonishing high viewing rate. (Beijing Youth Newspaper, 10-23-2008)

\section{(4) Makes it abnormal, hardly say anything due to being shocked.}

k. Her new hire style makes her be seen differently by her acquaints even though there was possibility that they were Lei-dao (shocked). (Beijing Youth Newspaper, 1116-2008)

1. The style are very untraditional which conformed to the astonishing (Lei-ren) effect described by producer. (Beijing Youth Newspaper, 11-16-2008)

m. The abnormal (Lei-ren) figure style with untidy clothing was widely criticized. (Beijing Youth Paper, 1229-2008)

\section{(5) Bad impression or makes people feel uncomfortable. It is a derogatory term.}

n. Lin Jiaxin would scare people to death in this weird fashion which is not appropriate even as casual wearing. It was believed that even the elders would not like to wear at company annually celebration. We strongly suggested to have style designer for her. (Beijing Youth Newspaper, 11-14-2008)

o. 'The various characters of the very bests of college entrance exam' has become the hot topic on Maopu website. It stemmed from an article titled 'the astonishing (Lei-si-ren) appearances of the very bests in 2008 college entrance exam' put on the website. In this article ten photos of the best students from different provinces were published. The appearances of these students were regarded as 'shocking and astonishing' (Lei-si-ren). (Southern Municipal Newspaper, 07-17-2008)

p. The figure in the photo looks astonishing (Lei), but the image quality is very high with excellent effect. (Beijing Youth Newspaper, 01-06-2008)

\section{(6) Beyond all expectations (only for joking). These are Neutral terms.}

q. 'Dating with girl is characterized of technique.' ..., this funny (Lei-ren-de) words slipped out from his month. (Beijing Youth Newspaper, 01-06-2009)

r. When got exam place, I was shocked (Lei-zhao-le). My goodness, the participants were so interesting. Some of them could be mothers, while some of them looked young children. (Beijing Youth Newspaper, 12-15-2008)

The meanings of 'Lei' family words mainly includes above six varieties. Some of them emphasizes shocking effect for subjects, while some of them stresses being shocked incidentally for objects. They can be used as verbs, adjectives, and noun. Because the uncertainty of their word characterizations, and the ambiguousness of their intensions and extensions, it looks difficult to correctly define their meanings. However, the view point of the main stream believes that since these words were exaggeratingly used in most cases, it is not necessary for them to have exact meanings. In many circumstances, we can only understand the right meaning based on the context.

As a root word, lei is of large derivation capability. For example, it can constitute neutral noun, like shocking people (Lei-ren), funny invitation (Lei-tie), interesting article (Lei-wen), amazing performance (Lei-ju), astonishing words (Lei-ci), abnormal professional (Leida-ren), and astonishing culture (Lei-wen-hua). It can also compose VC phrase, such as Lei-fan, Lei-dao, Leizhong, and modifying adjective, like Hen-lei, Zhui-lei . In addition, using rhetoric simulation method it may mimic catch phases appearing in TV and advertisement to become a sentence, and independently connect to other language units, like, we do not have to inform you before doing shocking (Lei-ni-mei-shang-liang), shocking people to the end (jiang-lei-ren-jin-xing-dao-di), no most astonishing, but more astonishing (mei-you-zui-lei, zhiyou-geng-lei), were you shocked today? (jin-tian-ni-beilei-dao-le-ma? ), the talk will never end, if there is not shocking effect (yu-bu-lei-ren-si-bu-xiu), there is not compensation if you are shocked to death (Lei-si-ren-buchang-ming), and many more.

Due to its wide acceptation in websites, Lei family phases has become more and more popular and generalized. Both paper based and audio and video based media are full of Lei family words. All human feelings which make people uncomfortable or shocked due to bad or good reason can be expressed with Lei or Lei-dao-le. Even meanings, like defeat, criticize, temptation, can also be 
expressed by Lei, which is regarded as extension of Lei family phase, for example,

Lei for defeat or beat:

s. The king of shocking (Lei-dao-tian-wang): Murray from English defeated defending champion Federer by

2:1 (Beijing Youth Newspaper, 11-28-2008)

Lei for blame or criticize:

t. With the shocking wave ( "lei" sheng) of "Xue Baocai becomes legend of white snake' (Beijing Youth Newspaper, 07-28-2008)

\section{Lei for messy:}

u. Short articles are characterized of Quan's style of 'the talk will never end if there is not shocking effect': do not fall in love with old man if he is not old enough. (Beijing Youth Newspaper, 12-01-2008)

Lei for show off, enticement:

v. (Her fashion clothing from different countries) constantly catch (Lei-zhao) people's eyes and soul in that little northern town. (Beijing Youth Newspaper, 12-022008)

\section{The reasons and pragmatic value of Lei occurrences}

The appearing of Lei family phases is inherently related to the original meaning of Lei which refers to sudden loud noise and exploding weapon, both of which imply strength and killing power, and further indicate mightiness or big bang. Through comparison we can see the common ground between Lei's original meaning and the meaning of catch word Lei. There exist perceptual interlinking base between the sudden loud noise of Lei and the feeling of shocking, astonishing brought up by event or material. That is these phases, like Lei, Lei-ren , Hen-lei , Lei-ju , Lei-dao , themselves are characterized of Lei which indicates thunder. Furthermore, the morphemes of ming, ting, bao in some groups of Lei based words, such as Lei-ming, Lei ting, Lei-dian, Leibao , also contain senses of big bang, blast, violence which are close to Lei in terms of meaning. These morphemes work together to produce people's psychological feeling of being hit by thunder and being shocked by electricity. And the further imagination helps to come out a series of brand new Lei family phrases.

The pragmatic Value of Lei family phrases

Language is the reflection of social life. It reflects people's current cultural psychology and social spirit. Young people are lively thinkers who tend to pursue new fashion, different life style, and fast path. Under the driving force of this kind of psychology, a batch of novel, expressive phrases as well as word combinations, including Lei family phrases, appear on Internet websites. It satisfied the Internet people who seek to be different, and are unorthodox. Some everyday words lost their freshness and concrete expression due to high frequency of use. The young people hope that they could be noticed by employing their own fresh words, and intently keep their specific and independent style. Lei family phrases are just able to suit this purpose. Both shocking (Lei-ren and being shocked (Bei-ren-lei) would create sensational effect. It may be said that Lei is an identity symbol for youth. Out of rebelling psychology to stereotypical language, lei has functionality to exaggeratingly convey the ironic sense of message, and is characterized of joking and humor.

Lei may be used to express the feeling status of people and produce vivid effect. One Chinese character may include multiple meanings, and the vivid Chinese character tends to invoke people's imagination. Even the pronunciation of a character would establish a connection to other homophones. The rich expression on Internet just came from traditional cultural thinking model and exploration of intrinsic capability of Chinese character. Since people are not able to see each other on the Internet, using expression symbol can be a creative format of web language. Lei is of vivid highlight effect in terms of audio as well as video. It is well competent for expression of swift feeling status and produces impact force in that aspects.

Lei has quite strong exclusiveness. Like other family phrases, Lei family phrases are of prominent population characteristics. The number of people who use them is limited. The users are most students and web surfers. It needs agreement between readers and writers at the same cultural level. That's why there exists exclusiveness for these kind of family phrases. Few people outside the group will understand the real meaning.

\section{Conclusion}

Catchword is a social phenomenon. Its popularity will not necessary go too long. Its biggest glamour lays in prevalence, freshness, uniqueness, or hard to understand. Some catchwords disappear very soon. But they were used and in prevalence ever. As a cultural phenomenon it is hard to be eliminated completely whatever it is positive or negative. We do not have to make fuss of the appearing of Lei family phrases whatever they are reasonable or unreasonable. After all they will surely be constrained by the natural path of language development. They either are accepted by more and more people and finally become catchwords, or disappear from our sight with time going by.

\section{References}

[1]. Yang, Zhixiang, Coexistence and pragmatic transmutation in contemporary Chinese. Fudan press, 2001

[2]. "Lei culture", Forward or backward of network language? Jiafang daily, 11-15-2008

[3]. Pan, Ruichun, Analysis of "zai nu", Study on rhetoric. 2008 (2) 